\title{
Morphometric Analysis of Mouse Airways After Chronic Allergen Challenge
}

\author{
Russ Ellis, Richard Leigh, David Southam, Paul M. O’Byrne, and Mark D. Inman \\ Firestone Institute for Respiratory Health and Department of Medicine, St. Joseph's Healthcare and McMaster \\ University, Hamilton, Ontario, Canada
}

SUMMARY: Understanding the mechanisms of airway remodeling in chronic allergic conditions such as asthma is increasingly dependent on the use of animal models. Techniques for quantifying structural changes are required that are reproducible and responsive and that can be applied to different staining techniques in both human and animal airway tissues. Here, we characterize a morphometric technique to quantify changes in extracellular matrix and contractile tissue as two indices of airway remodeling in mice. Specific aims were to establish the optimum projection beneath the epithelium to assess remodeling changes and to determine whether such changes are reproducible within different areas of the lung. Finally, based on the variance within measurements, we calculated sample size requirements for research applications of this technique. BALB/c mice were sensitized to ovalbumin and studied after chronic allergen challenge. Lungs were formalin fixed and sectioned were then assayed for extracellular matrix or contractile tissue using morphometric/colorimetric techniques. In this model, the optimum projected distance to measure changes in extracellular matrix or contractile tissue was $20 \mu \mathrm{m}$ beneath the epithelium; projecting beyond this depth resulted in decreased ability to detect allergen-induced changes (signal) because of increased irrelevant staining of surrounding parenchymal tissue (noise). The technique was responsive, because an allergen-induced signal was detected in all airway sections and all lung regions studied $(p<0.05)$. The power of this analysis was such that allergen-induced changes can be reliably ( $>80 \%$ power) detected using 8 to 10 mice. This morphometric technique provides a valid and objective method to assess structural changes in the airways of mice after chronic allergen exposure. (Lab Invest 2003, 83:1285-1291).

\begin{abstract}
A sthma is characterized by the presence of variable airflow limitation, airway hyper-responsiveness, and chronic airway inflammation. However, mechanisms underlying the physiologic airway dysfunction remain unclear. Bronchoscopy studies have demonstrated that structural changes are present in asthma (Jeffery et al, 1989), even in the airways of symptomatically mild patients (Chetta et al, 1997). These changes, often grouped under the heading "airway remodeling," include increased smooth muscle (hyperplasia or hypertrophy), thickening of the airway wall (associated with epithelial and goblet cell hyperplasia), myofibroblast hyperplasia, and subepithelial fibrosis (Carroll et al, 1993; Dunnil, 1960; Jeffery et al, 1989, 1992; Roche et al, 1989). Observations that the extent of fibrotic changes are related to the degree of airway eosinophilia (Chetta et al, 1996) and an increase in patients with allergic rhinitis who progress to asthma (Boulet et al, 2000) support the concept that at least some of the remodeling changes are progressive in response to an underlying inflammatory process. Furthermore, findings that the extent of fibrotic changes
\end{abstract}

DOI: 10.1097/01.LAB.0000087586.25982.B5

Received June 3, 2003.

This study was funded in part by the Canadian Institutes for Health Research and the Ontario Thoracic Society.

Address reprint requests to: Dr. M. D. Inman, Associate Professor of Medicine, Firestone Institute for Respiratory Health, St. Joseph's Healthcare, 50 Charlton Avenue East, Hamilton, Ontario, Canada L8N 4 A6.

E-mail:inmanma@mcmaster.ca are related to airway hyperresponsiveness (Boulet et al, 1997; Jeffery et al, 1989) and asthma symptoms (Chetta et al, 1997) support the concept that these structural changes play a role in the underlying airway dysfunction in asthma.

Observations that indices of remodeling are present even in newly diagnosed asthma (Chetta et al, 1997) have made it difficult to study the development or functional importance of specific airway remodeling events. For this reason, many investigators have turned to animal models, in which periods of chronic allergen challenge have produced various markers of airway remodeling with an associated airway hyperresponsiveness (Bai et al, 1995; Blease et al, 2001; Palmans et al, 2000; Salmon et al, 1999; Temelkovski et al, 1998). Of these models, those based in mice (Blease et al, 2001; Temelkovski et al, 1998) are particularly attractive given the availability of blocking antibodies and gene-deficient and transgenic mice that allow for mechanistic studies. To study the mechanisms of the remodeling processes and the relationship between resulting structural changes and airway dysfunction, it is crucial that methods used to quantify these structural changes are well characterized, reproducible, and responsive. In the case of assaying the extent of subepithelial fibrosis, methods that have been used in the past include subjective description of the extent of peribronchial collagen staining (Blease et al, 2002), semiquantitative description of the same stain (ie, 0-4 scale) (Henderson et al, 2002), quantification of total lung collagen or hydroxyproline (Lee et 
al, 2001; Sime et al, 1997), and morphometric assessment of specific matrix stains (Lee et al, 2001; Leigh et al, 2002). Changes in peribronchial contractile tissue staining have also been assessed using subjective (Yamamoto et al, 2003) and morphometric (Leigh et al, 2002; Moir et al, 2003) methods. Although each of these methods have demonstrated changes in fibrotic or contractile tissue in response to specific interventions, there is minimal information available indicating how these techniques may be used optimally to detect such changes in the mouse airway wall.

The purpose of the current study was to fully characterize a previously used morphometric method (Leigh et al, 2002) for quantifying subepithelial fibrosis and contractile tissue in mice airways after chronic allergen challenge. This technique combined computerized morphometric and colorimetric techniques to quantify the area positive for Masson's Trichrome (MT) stain or $\alpha$-smooth muscle actin ( $\alpha \mathrm{SMA})$ stain within a given depth beneath the epithelium of the first generation airway. The specific hypothesis addressed in this study was that the power of this technique would be influenced by the depth beneath the epithelium that was analyzed for the presence of each specific stain. We also examined whether detection of allergeninduced changes in these measurements was consistent in several lung regions.

\section{Results}

The mean airway ratios for major to minor axes for all tissue segments are shown in Table 1. No differences were detected between saline and allergenchallenged mice $(p>0.05)$ nor were there differences detected in the ratios between the superior and inferior lung segments from the left lung $(p>0.05)$. The mean ratio of all sections obtained from the right lung (2.72: SD 1.04) was significantly greater than that for all sections obtained from the left lung (1.19: SD 0.11) $(p<$ 0.05).

\section{What Is the Optimal Depth Beneath the Airway Epithelium to Assess Allergen-Induced Fibrotic Changes?}

Significant differences in percent MT staining were detected between the saline (SAL) and ovalbumin

Table 1. Airway Ratios (Mean and SD) of the First Generation Airway for Each Lobe Segment from SAL and OVA Tissue

\begin{tabular}{clc}
\hline Group & \multicolumn{1}{c}{ Lobe } & $\begin{array}{l}\text { Airway ratio } \\
\text { [mean (SD)] }\end{array}$ \\
\hline \multirow{2}{*}{ SAL } & Right inferior & $2.46(1.07)$ \\
& Left superior & $1.24(0.11)$ \\
\multirow{2}{*}{ OVA } & Left inferior & $1.17(0.10)$ \\
& Right inferior & $2.98(1.01)$ \\
& Left superior & $1.20(0.13)$ \\
& Left inferior & $1.16(0.09)$ \\
\hline
\end{tabular}

Ratios calculated as maximum diameter $(\mu \mathrm{m})$ divided by minimum diameter $(\mu \mathrm{m})$.

SAL, saline; OVA, ovalbumin.
(OVA) tissue using expansion values of 10, 20, 30, 40, and $50 \mu \mathrm{m}$ beneath the basal epithelium $(p<0.05)$ but not values of 60 or $70 \mu \mathrm{m}$ (Fig. 1A). The greatest magnitude of difference between saline and OVAchallenged mice was 5.49 percentage units, observed using a $20-\mu \mathrm{m}$ expansion. Sample sizes required to detect differences between the SAL and OVA tissue ( $\beta$ $=0.20$ ) were calculated for each expansion value. The smallest sample size required was 8 mice, using an expansion value of $20 \mu \mathrm{m}$, and ranged from 10 to 16 for the other values examined. For the $\alpha$ SMA stain, significant differences were detected using expansion values of 20,30 , and $40 \mu \mathrm{m}(p<0.05)$ but not values of $10,50,60$, or $70 \mu \mathrm{m}$ (Fig. 1B). The greatest magnitude of difference between saline and OVAchallenged mice was 7.78 percentage units, observed using a $20-\mu \mathrm{m}$ expansion. Sample sizes required to detect differences between the SAL and OVA tissue $(\beta$ $=0.20$ ) were calculated for each expansion value. The smallest sample size required was 10 mice, using an expansion value of $20 \mu \mathrm{m}$, and ranged from 14 to 28 for the other values examined.

\section{Are Allergen-Induced Changes in Lung Structure Detected Within Different Lobes or Regions of the Lung?}

Significant differences in percent MT staining between SAL and OVA tissue, using a 20- $\mu \mathrm{m}$ expansion, were detected in each of the three lung segments we studied ( $p<0.05$ ) (Fig. 2). The magnitude of difference was larger in the left lung samples (5.17-5.49 percentage units) compared with the right lung samples (4.41

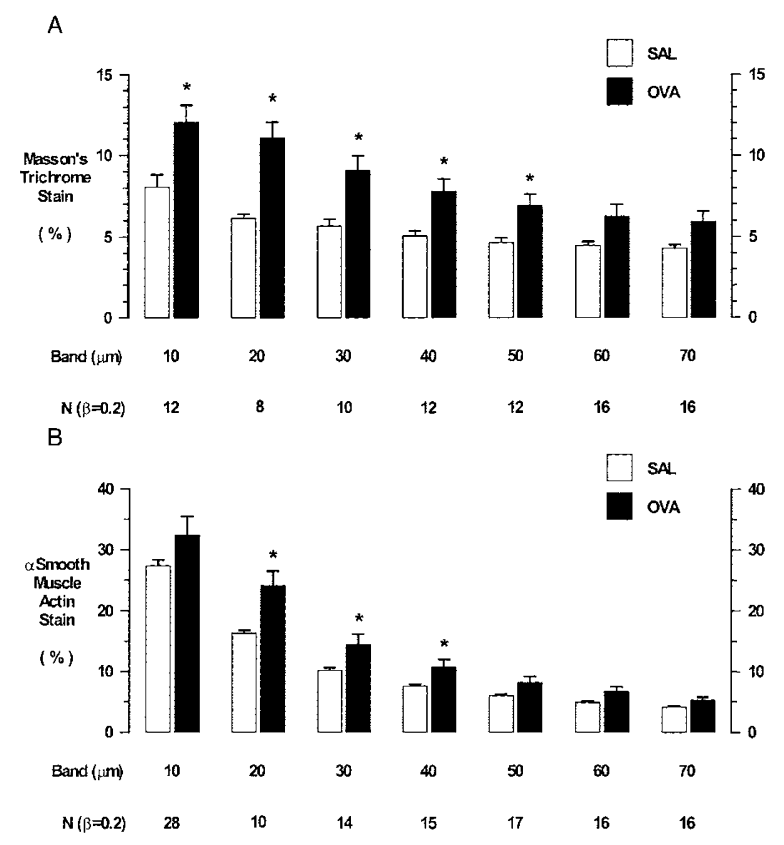

Figure 1.

Quantification of the extent of Masson's Trichrome (MT) staining (A) or $\alpha$-smooth muscle actin staining ( $\alpha$ SMA) (B) in SAL- and OVA-challenged lung tissue when the projected distance from the basement membrane is increased incrementally from 10 to $70 \mu \mathrm{m}$. Sample sizes $N(\beta=0.20)$ were calculated as the number of mice required to detect differences between the SAL and OVA tissue. 

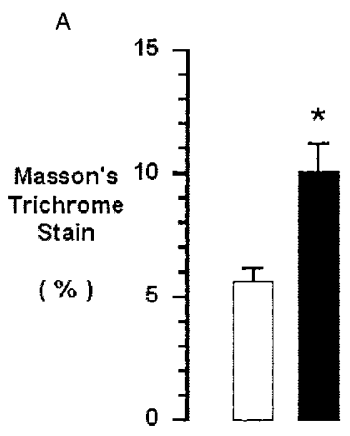

$N(\beta=0.2)$

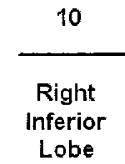

B
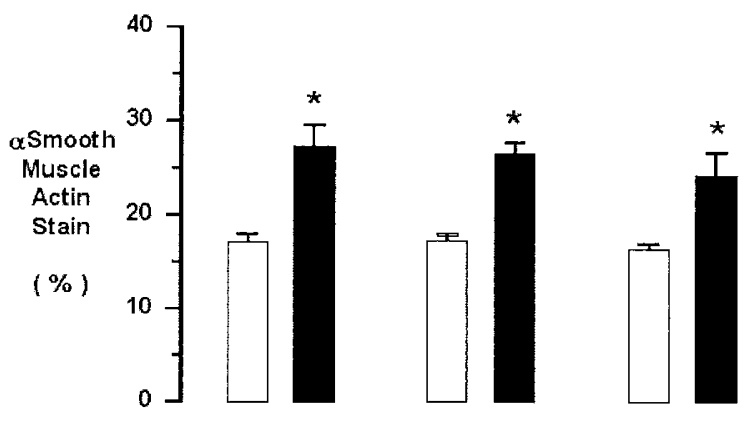

$N(\beta=0.2)$
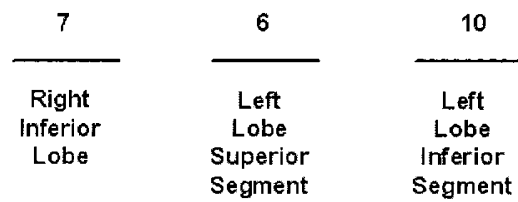

Figure 2.

Quantification of the extent of MT staining (A) or $\alpha$ SMA staining (B) in SAL and OVA-challenged lung tissue in single sections from tissue obtained from the right inferior lobe and from the left lobe bisected into superior and inferior segments. Sample sizes $N(\beta=0.20)$ were calculated as the number of mice required to detect differences between the SAL and OVA tissue.

percentage units). Sample sizes required to detect differences between the SAL and OVA tissue $(\beta=$ $0.20)$ were calculated for each lung segment. Both superior and inferior left lobe segments required a sample size of 8 mice to detect differences, whereas the right inferior lobe required a sample size of 10 mice. For the $\alpha$ SMA stain, significant differences were also detected in each of the three lung segments $p<$ 0.05) (Fig. 2B). Sample sizes required to detect differences between the SAL and OVA tissue $(\beta=0.20)$ were calculated for each lung segment. The left superior lobe required a sample size of 6 mice, the left inferior lobe required 10 mice, and the right inferior lobe required 7 mice.

There were no differences in parenchymal staining between the SAL and OVA groups for each stain. For MT staining, SAL tissue was $0.45 \%$ (SEM 0.12) compared with $0.37 \%$ (SEM 0.15) for OVA $(p>0.05)$. For $\alpha$ SMA staining, SAL tissue was $1.51 \%$ (SEM 0.11) compared with $2.08 \%$ (SEM 0.24) for OVA ( $p>0.05$ ).

\section{Discussion}

The results of this study indicate that this morphometric method is a feasible, valid, and reliable technique to assess subepithelial fibrosis and contractile tissue remodeling in a murine model of chronic allergeninduced airway inflammation. Here we have demonstrated that the power to detect allergen-induced fibrotic and contractile remodeling is influenced by the depth beneath the epithelium that is analyzed for morphometric changes. Moreover, we have observed that these changes seem to occur uniformly in the middle third of the left lung and are also observed in the right lung. This information should be extremely useful to reduce animal numbers in future studies, and to reduce type II errors, in which allergen-induced remodeling might be missed because of poor sampling techniques.

Validity of a test is determined by its ability to consistently detect a true positive signal. Using our current method in our model of chronic allergen challenge, we were able to detect allergen-induced changes in randomly selected sections from three different regions of the left or right lung. The range of expansion depths at which we were able to detect allergen-induced changes in MT staining ranged from 10 to $50 \mu \mathrm{m}$ beneath the airway epithelium; the maximal difference was evident at a depth of $20 \mu \mathrm{m}$. At expansion depths $>50 \mathrm{~mm}$, differences between OVA- and SAL-challenged mice were no longer apparent and likely reflect a decrease in the signal to noise ratio caused by the inclusion of underlying parenchymal tissue. To detect $\alpha$ SMA staining differences between saline and allergen-challenged mice required expansion depths of 20,30 , or $40 \mu \mathrm{m}$, with maximal differences also detected at $20 \mu \mathrm{m}$. Our results indicate that this morphometric analytic technique is a valid method for assessing remodeling of subepithelial fibrotic and contractile tissue in the airway wall. These observations also reflect a feature of this model, namely that fibrotic and contractile tissue changes are restricted to the peribronchial region and do not include lung parenchyma.

A concern when identifying pathologic changes in airway models of chronic inflammation is where in the lung to best locate these changes. Here, we propose that the first generation airway in the middle third of the left lobe should be used for such investigations. The reason for this recommendation is 2-fold. First, this approach allows the investigator to retain a high degree of objectivity in terms of selecting airways for analysis, while ensuring that the airways being compared within each group are of the same generation. Second, we observed that there were few visually obvious changes in the structure of smaller airways. Clearly this latter observation may be influenced by the method of delivering allergen. Our intranasal delivery method is based on aspiration (Southam et al, 2002), resulting in distribution to proximal airways, 
whereas inhalation delivery methods (Ohkawara et al, 1997) would likely result in more peripheral distribution and correspondingly different pathologic changes than observed here.

Our results indicate that a consistently tangential orientation of the airway was obtained when analyzing the left lung and that allergen-induced changes were similar in this lung when we obtained sections from the extreme superior and inferior borders of the middle third of this lung. This demonstrates a considerable degree of latitude for researchers aiming to detect and quantify allergen-induced remodeling changes in similar models.

For appropriate ethical reasons, funding agencies and institutional review boards increasingly require that investigators demonstrate, a priori, that their studies are sufficiently powered $(>80 \%)$ to detect the primary outcome of interest. Based on power calculations, the results of our study indicate that using an expansion depth of $20 \mu \mathrm{m}$ requires the fewest number of mice (8-10 per group) to detect a significant difference in the degree of subepithelial fibrosis or contractile remodeling between SAL control and OVAchallenged mice.

In conclusion, we have described a technique of airway morphometry that is valid and reliable for quantifying the amount of peribronchial extracellular matrix and contractile tissue in a mouse model of chronic airway inflammation. The technique allows investigators to objectively quantify morphologic changes in mouse airways, thereby minimizing investigator bias associated with many of the more subjective techniques currently in use. Clearly, when authors use challenge models that are substantially different from ours, the depth at which remodeling changes occur may differ. In such cases, we strongly recommend that an approach similar to that described here be used to optimize the potential for detecting allergen-induced effects.

\section{Materials and Methods}

\section{Animals}

Female BALB/c mice, age 10 weeks at study onset, were housed in specific pathogen-free conditions. All procedures were reviewed and approved by the Animal Research Ethics Board at McMaster University and conformed to National Institutes of Health guidelines (Washington DCUSGPO, 1985).

\section{Sensitization and Challenge}

As in the past, we sensitized mice with intraperitoneal ovalbumin (Sigma, Oakville, Ontario, Canada) conjugated to aluminum potassium sulfate (IP OVA) injected on Days 1 and 11 and intranasal ovalbumin (IN OVA) on Day 11 (Inman et al, 1999). Sensitized mice were subjected to chronic periods of allergen exposure, which involved six 2-day periods of IN OVA challenges, each separated by 12 days (10 weeks total). Control mice were sensitized with OVA but received saline (SAL) challenges. Mice ( $n=9$ SAL, 10 OVA) were studied at 4 weeks after the final challenge (Fig. 3).

\section{Lung Histologic Examination and Morphometry}

Dissected lungs were inflated with $10 \%$ buffered formalin to a pressure of $20 \mathrm{cmH}_{2} \mathrm{O}$, ligated at the trachea, and then both lungs were fixed in formalin for 24 hours. After fixation, the right inferior lobe and the left lobe were isolated and bisected into superior and inferior segments (Fig. 4A). After histologic processing

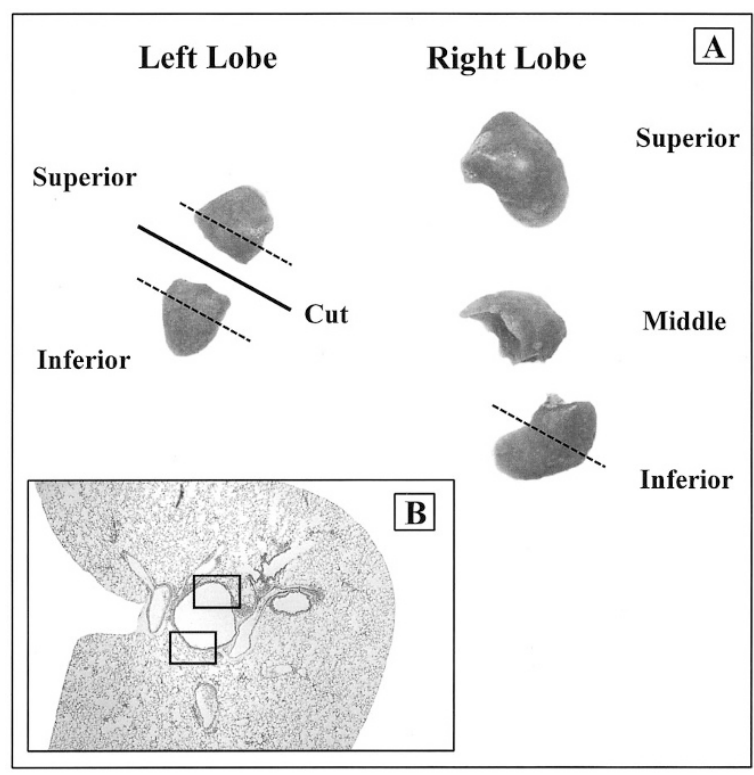

Figure 4.

A, Depiction of the lobes of a mouse lung after inflation and fixation with formalin. The left lobe and right inferior lobe were bisected. Sections for staining were obtained from the exposed surfaces of the right lobe segments, and from regions corresponding to the extreme inferior and superior borders of the middle third of the left lobe (all sections indicated by dashed lines). B, Typical first generation airway from the left lobe with associated artery and vein. Airway images captured for analysis indicated in boxed regions.

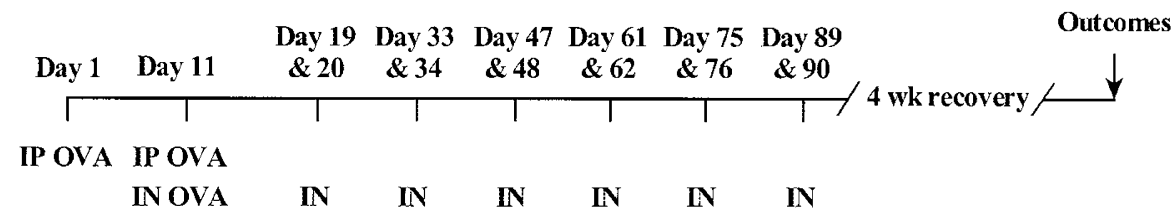

Figure 3.

Sensitization and chronic challenge protocol. Lung tissues were collected after a 4-week recovery period following the final challenge. 
(Leigh et al, 2002), both segments of the right inferior lobe were embedded together in paraffin, whereas the superior and inferior segments of the left lobe were embedded in individual paraffin blocks. All tissues were embedded with the sectioned face down and were orientated to obtain transverse cross-sections of the first generation airway (Fig. 4B). This airway is often macroscopically visible in the left lobe before embedding but less so in the right lobe. For this reason, the left lobe segments were embedded separately and the right inferior lobe segments embedded together to ensure good airway representation.

The embedded tissues from the left lobe were rough cut to approximately half the original size, whereas the right lobe segments were rough cut to only expose a smooth tissue surface. Three-micron-thick transverse sections were then cut and stained with either MT to demonstrate extracellular matrix or $\alpha \mathrm{SMA}$ (clone NCLSMA; Novocastra Laboratories Ltd., Newcastle, United Kingdom) to identify contractile elements. For all tissues, the first generation airway was identified while viewing under a microscope at $\times 4$ magnification (Olympus BX40; Carsen Group Inc., Markham, Ontario, Canada), and the major and minor axis were measured with a customized digital image analysis system (Northern Eclipse, version 6; Empix Imaging Inc., Mississauga, Ontario, Canada) using a digital pen mouse and drawing tablet. The ratio of the diameters (maximum/minimum) was calculated as a reflection of the degree to which the sectioning of the airway was at right angles to its long axis.

Morphometric/colorimetric quantification involved calculating the percentage of defined areas of tissue that were positively stained using the same customized digital image analysis system. While viewing under the microscope at $\times 20$ magnification, images of the entire first generation airway wall that were free of intimate contact with the neighboring vessels (Fig. 5A) were captured into the computer by an investigator blinded to the tissue codes. Analysis was initiated by tracing a line along the basal border of the epithelium using the digital pen mouse and drawing tablet. This line did not cover any stained tissue of interest. Thereafter, the software macro application projected a second line in a direction away from the center of the airway to create a band of tissue for colorimetric analysis. The width of the band was variable and predetermined by the investigator, allowing for repeated analysis on a single image (Fig. 5B). The software then calculated the percentage of each band that was positively stained, based on previously determined color plane settings. Thus, final percent staining scores were obtained for each mouse for both stains of interest. Analytic approaches were aimed at addressing two primary questions:

1. What is the optimal depth beneath the airway epithelium to assess allergen-induced fibrotic changes?

Bands of tissue, with expansion depths ranging from 10 to $70 \mu \mathrm{m}$ measured in $10-\mu \mathrm{m}$ increments, were obtained from the inferior segment of the left lung (Fig. 5B). Comparisons were made of percent MT staining or percent $\alpha \mathrm{SMA}$ staining between SAL and OVA-challenged mice. To assess which band depth is optimal for performing comparative studies, power calculations were performed on these data, with the results expressed as the number of mice required to demonstrate allergen-induced changes in either stain of interest.

2. Are allergen-induced changes in lung structure detected within different lobes or regions of the lung?

The percent MT stain or percent $\alpha$ SMA stain was compared between SAL and OVA-challenged mice using data obtained from a $20-\mu \mathrm{m}$ subepithelial band from the first generation airway of the inferior and superior segments of the left lobe and inferior right lobe. Power calculations were performed on these data, with the results expressed as the number of mice required to demonstrate allergen-induced changes in either stain of interest for each lung section.
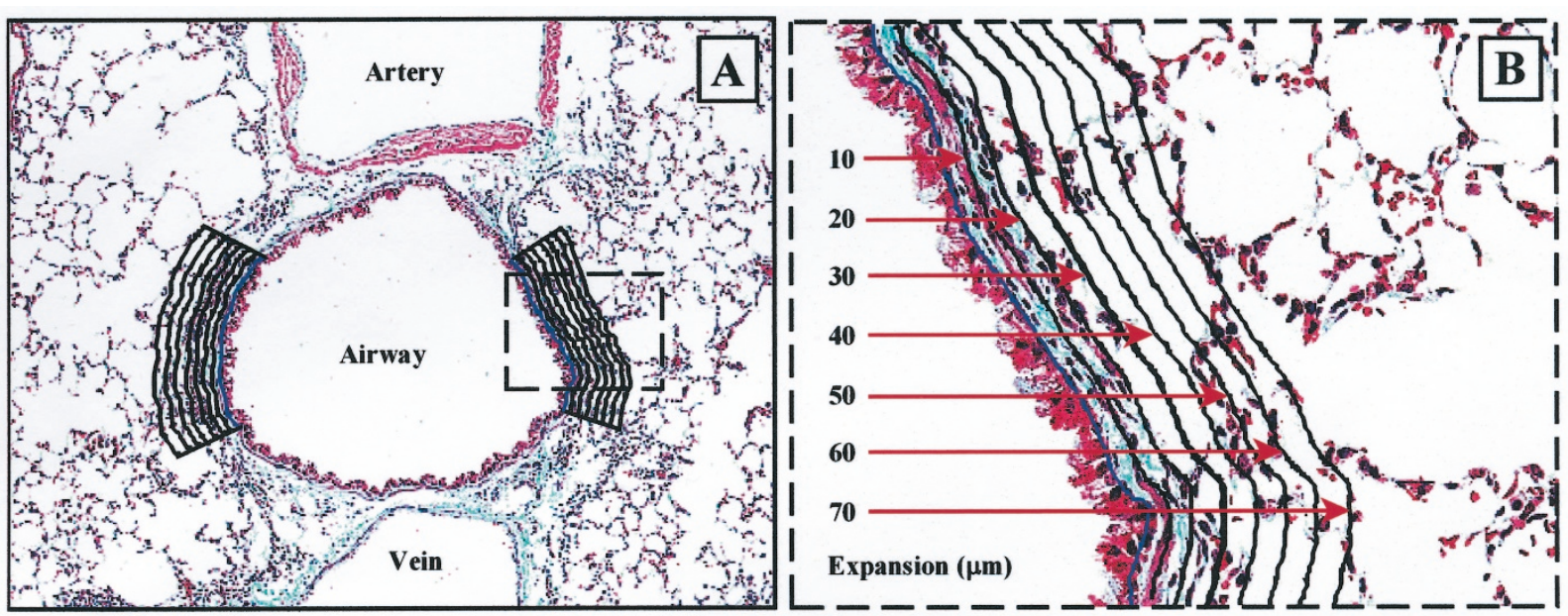

Figure 5.

A, Depiction of first generation airway captured for analysis. The airway is associated with an artery and vein. B, The basal border of the epithelium is traced by hand (blue line) then projected by software into the surrounding parenchyma (black lines), a distance determined by the operator. The stain of interest (MT) is green and is quantified by the software as a percentage of the total band area. 
To determine that parenchymal staining was equal between SAL and OVA tissues and did not have a systematic influence on morphometric analysis of the airway, bands of parenchymal tissue from the left inferior lobe for both stains were analyzed. A 20- $\mu \mathrm{m}$ band located a distance between 20 and $40 \mu \mathrm{m}$ from the border of the epithelium was captured for analysis. This band was composed primarily of parenchyma, and percent staining of MT or $\alpha$ SMA was analyzed from both SAL and OVA groups.

\section{Statistical Analysis}

Data on all figures are expressed as mean and SEM. Descriptive data in Table 1 are expressed as mean and SD. All comparisons between saline control mice and mice receiving chronic allergen exposure were made using Student's $t$ test. All comparisons were two-tailed assuming equal variance, and $p$ values $<$ 0.05 were considered to be significant. Sample-size requirements were based on a Student's $t$ test analysis and calculated with an assumed power of $80 \%$ and an $\alpha$ of 0.05 as we have done previously (Dahlen et al, 2001).

\section{Acknowledgements}

The authors thank Ms. Jennifer Wattie for technical assistance. Dr. Richard Leigh is a Canadian Institutes for Health Research Fellow. Dr. Mark Inman is the Harbinger Scholar in Respiratory Medicine.

\section{References}

Bai TR, Wang ZL, Walker B, and Pare PD (1995). Chronic allergic inflammation induces replication of airway smooth muscle cells in vivo in guinea pigs. Chest 107:93S.

Blease K, Jakubzick C, Westwick J, Lukacs N, Kunkel SL, and Hogaboam CM (2001). Therapeutic effect of IL-13 immunoneutralization during chronic experimental fungal asthma. J Immunol 166:5219-5224.

Blease K, Schuh JM, Jakubzick C, Lukacs NW, Kunkel SL, Joshi BH, Puri RK, Kaplan MH, and Hogaboam CM (2002). Stat6-deficient mice develop airway hyperresponsiveness and peribronchial fibrosis during chronic fungal asthma. Am J Pathol 160:481-490.

Boulet LP, Laviolette M, Turcotte H, Cartier A, Dugas M, Malo $\mathrm{JL}$, and Boutet M (1997). Bronchial subepithelial fibrosis correlates with airway responsiveness to methacholine. Chest 112:45-52.

Boulet LP, Turcotte H, Laviolette M, Naud F, Bernier MC, Martel S, and Chakir J (2000). Airway hyperresponsiveness, inflammation, and subepithelial collagen deposition in recently diagnosed versus long-standing mild asthma: Influence of inhaled corticosteroids. Am J Respir Crit Care Med 162:1308-1313.

Carroll N, Elliot J, and James A (1993). The structure of the large and small airways in nonfatal and fatal asthma. Am Rev Respir Dis 147:405-410.

Chetta A, Foresi A, Del Donno M, Bertorelli G, Pesci A, and Olivieri D (1997). Airways remodeling is a distinctive feature of asthma and is related to severity of disease. Chest 111:852857.
Chetta A, Foresi A, Del Donno M, Consigli GF, Bertorelli G, Pesci A, Barbee RA, and Olivieri D (1996). Bronchial responsiveness to distilled water and methacholine and its relationship to inflammation and remodeling of the airways in asthma. Am J Respir Crit Care Med 153:910-917.

Dahlen B, O'Byrne PM, Watson RM, Roquet A, Larsen F, and Inman MD (2001). The reproducibility and sample size requirements of exercise-induced bronchoconstriction measurements. Eur Respir J 17:581-588.

Dunnil MS (1960). The pathology of asthma with special reference to changes in the bronchial mucosa. J Clin Pathol 13:27-32.

Henderson WRJ, Tang L, Chu SJ, Tsao SM, Chiang GK, Jones F, Jonas M, Pae C, Wang H, and Chi EY (2002). A role for cysteinyl leukotrienes in airway remodeling in a mouse asthma model. Am J Respir Crit Care Med 165:108-116.

Inman MD, Ellis R, Wattie J, Denburg JA, and O'Byrne PM (1999). Allergen-induced increase in airway responsiveness, airway eosinophilia and bone marrow eosinophil progenitors in mice. Am J Respir Cell Mol Biol 21:473-479.

Jeffery PK, Godfrey RW, Adelroth E, Nelson F, Rogers A, and Johansson SA (1992). Effects of treatment on airway inflammation and thickening of basement membrane reticular collagen in asthma: A quantitative light and electron microscopic study. Am Rev Respir Dis 145:890-899.

Jeffery PK, Wardlaw AJ, Nelson FC, Collins JV, and Kay AB (1989). Bronchial biopsies in asthma: An ultrastructural, quantitative study and correlation with hyperreactivity. Am Rev Respir Dis 140:1745-1753.

Lee CG, Homer RJ, Zhu Z, Lanone S, Wang X, Koteliansky V, Shipley JM, Gotwals P, Noble P, Chen Q, Senior RM, and Elias JA (2001). Interleukin-13 induces tissue fibrosis by selectively stimulating and activating transforming growth factor beta(1). J Exp Med 194:809-821.

Leigh R, Ellis R, Wattie J, Southam DS, De Hoogh M, Gauldie J, O'Byrne PM, and Inman MD (2002). Dysfunction and remodeling of the mouse airway persist after resolution of acute allergen-induced airway inflammation. Am J Respir Cell Mol Biol 27:526-535.

Moir LM, Leung SY, Eynott PR, McVicker CG, Ward JP, Chung KF, and Hirst SJ (2003). Repeated allergen inhalation induces phenotypic modulation of smooth muscle in bronchioles of sensitized rats. American J Physiol Lung Cell Mol Physiol 284:L148-L159.

Ohkawara Y, Lei XF, Stampfli MR, Marshall JS, Xing Z, and Jordana M (1997). Cytokine and eosinophil responses in the lung, peripheral blood, and bone marrow compartments in a murine model of allergen-induced airways inflammation. Am J Respir Cell Mol Biol 16:510-520.

Palmans E, Kips JC, and Pauwels RA (2000). Prolonged allergen exposure induces structural airway changes in sensitized rats. Am J Respir Crit Care Med 161:627-635.

Roche WR, Beasley R, Williams JH, and Holgate ST (1989). Subepithelial fibrosis in the bronchi of asthmatics. Lancet $1: 520-524$.

Salmon M, Walsh DA, Koto $\mathrm{H}$, Barnes PJ, and Chung KF (1999). Repeated allergen exposure of sensitized BrownNorway rats induces airway cell DNA synthesis and remodelling. Eur Respir J 14:633-641. 
Sime PJ, Xing Z, Graham FL, Csaky KG, and Gauldie J (1997). Adenovector-mediated gene transfer of active transforming growth factor-beta1 induces prolonged severe fibrosis in rat lung. J Clin Invest 100:768-776.

Southam DS, Dolovich M, O'Byrne PM, and Inman MD (2002). Distribution of intranasal instillations in mice: Effects of volume, time, body position, and anesthesia. Am J Physiol Lung Cell Mol Physiol 282:L833-L839.

Temelkovski J, Hogan SP, Shepherd DP, Foster PS, and Kumar RK (1998). An improved murine model of asthma: Selective airway inflammation, epithelial lesions and increased methacholine responsiveness following chronic exposure to aerosolized allergen. Thorax 53:849-856.
Washington DCUSGPO (1985). Guide for the Care and Use of Laboratory Animals. National Institutes of Health Publication No. 86-23. Washington DC: U.S. Government Printing Office.

Yamamoto Y, Tanaka A, Kanamaru A, Tanaka S, Tsubone H, Atoji $Y$, and Suzuki $Y$ (2003). Morphology of aging lung in F344/N rat: Alveolar size, connective tissue, and smooth muscle cell markers. Anat Rec 272A:538-547. 